\title{
Build 3D Nanoparticles by Using Ultrathin 2D MOFs Nanosheets for NIR Light-Triggered Molecular Switching
}

Jia-Ying Wang, a,c Yang-Hui Luo *a,c, Feng-Hao Xing a , Xiao-Wei Jin a, Li-Hong Guo, ${ }^{b}$ Li-Hai Zhai, ${ }^{b}$ Lan Zhang, ${ }^{a}$ Wen-Xia Fang, ${ }^{a}$ and Bai-Wang Sun *a

a School of Chemistry and Chemical Engineering, Southeast University, Nanjing, 211189, P.R. China. E-mail: peluoyh@sina.com (LYH); chmsunbw@seu.edu.cn (SBW).

b Lunan Pharmaceutical Co. Ltd., Linyi 276000, Shandong, China.

c The authors are contributed equally. 


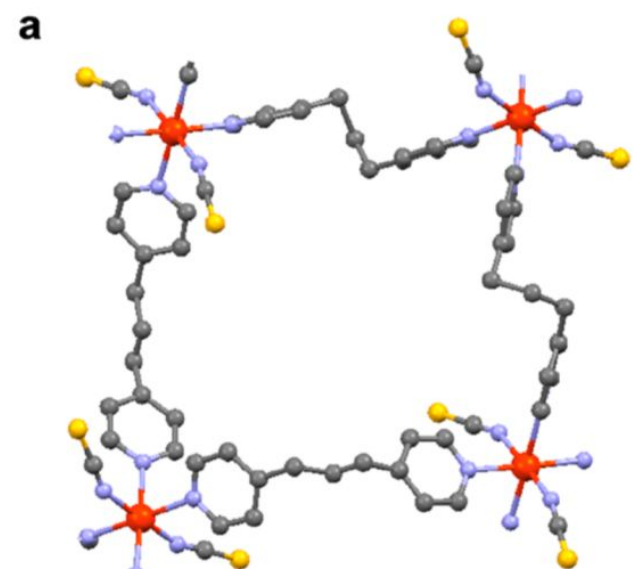

b

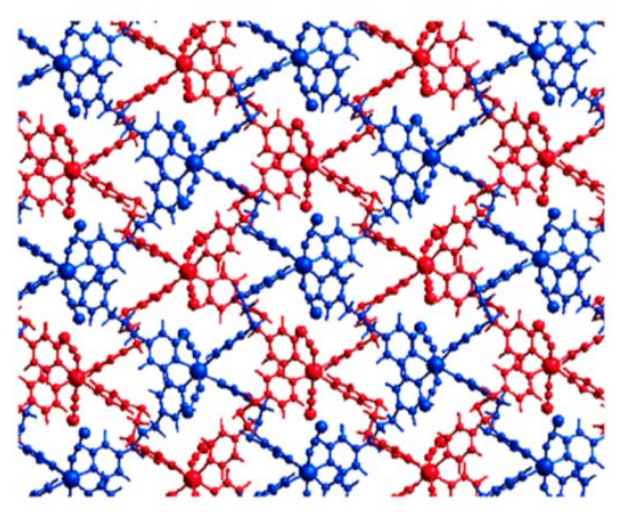

Figure S1. (a) connectivity style for a $(4,4) \mathrm{Fe}(1,3-\mathrm{bpp})_{2}$ rhombic grid unit; (b) topological views of the two-fold interpenetrated 2D sheets.

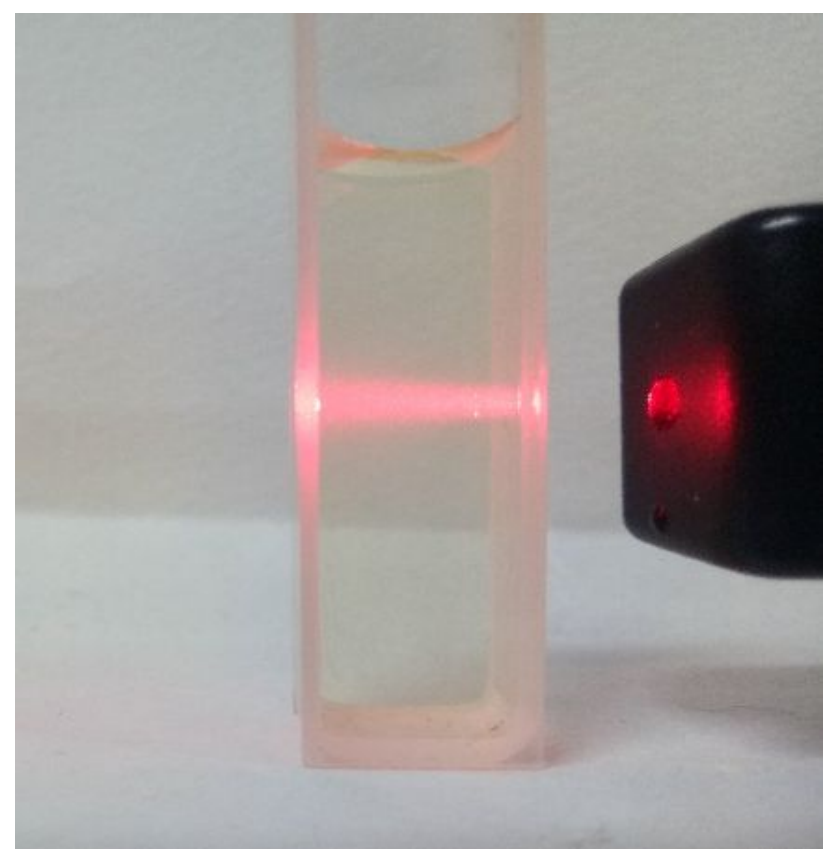

Figure S2. Tyndall effect of the 2D aqueous colloidal suspension. 


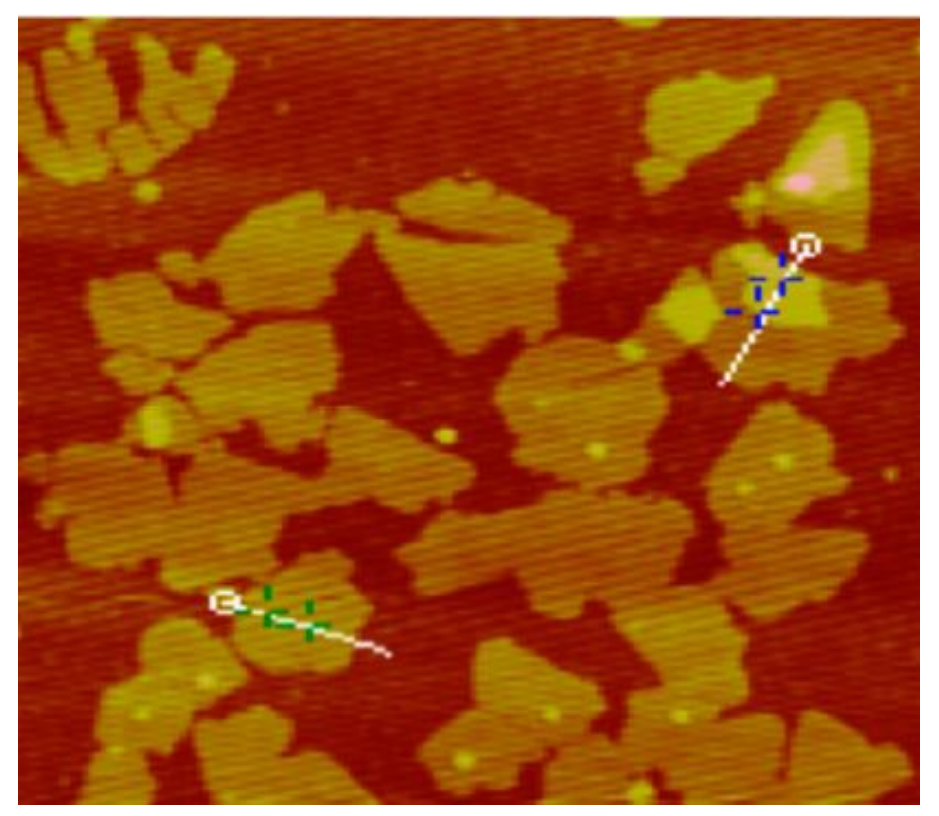

Figure S3. AFM topological image of 2D SCO nanosheets.

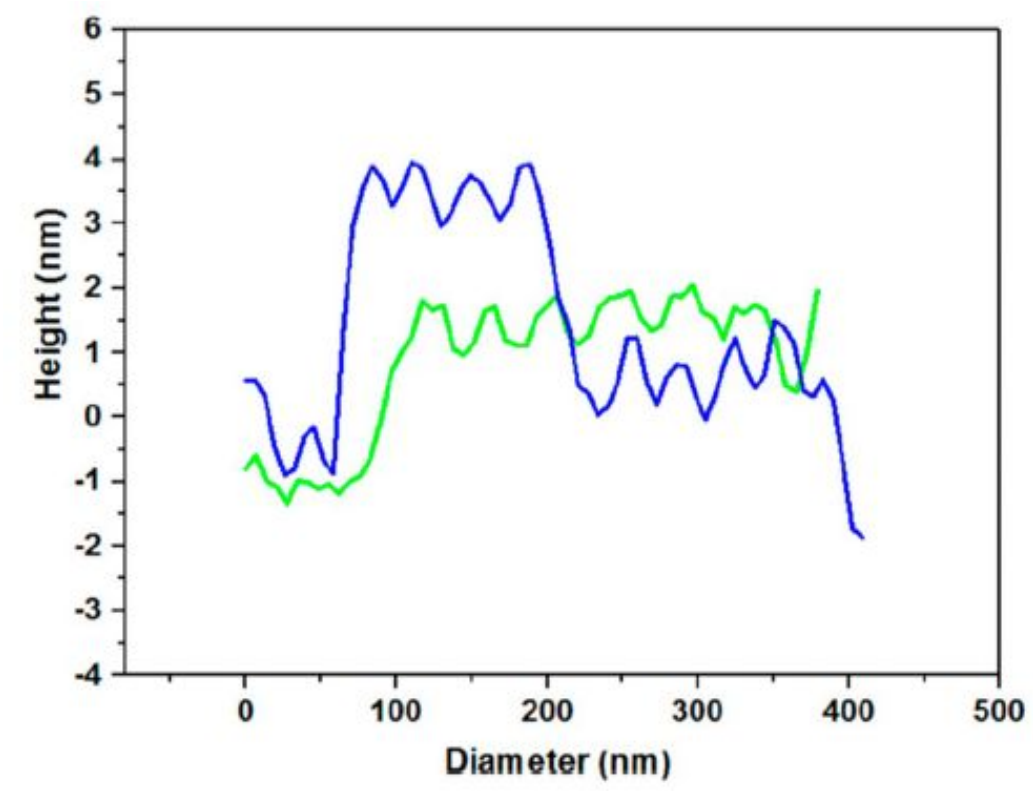

Figure S4. Height profile along the white lines for different regions for AFM topological image in Figure S3. 


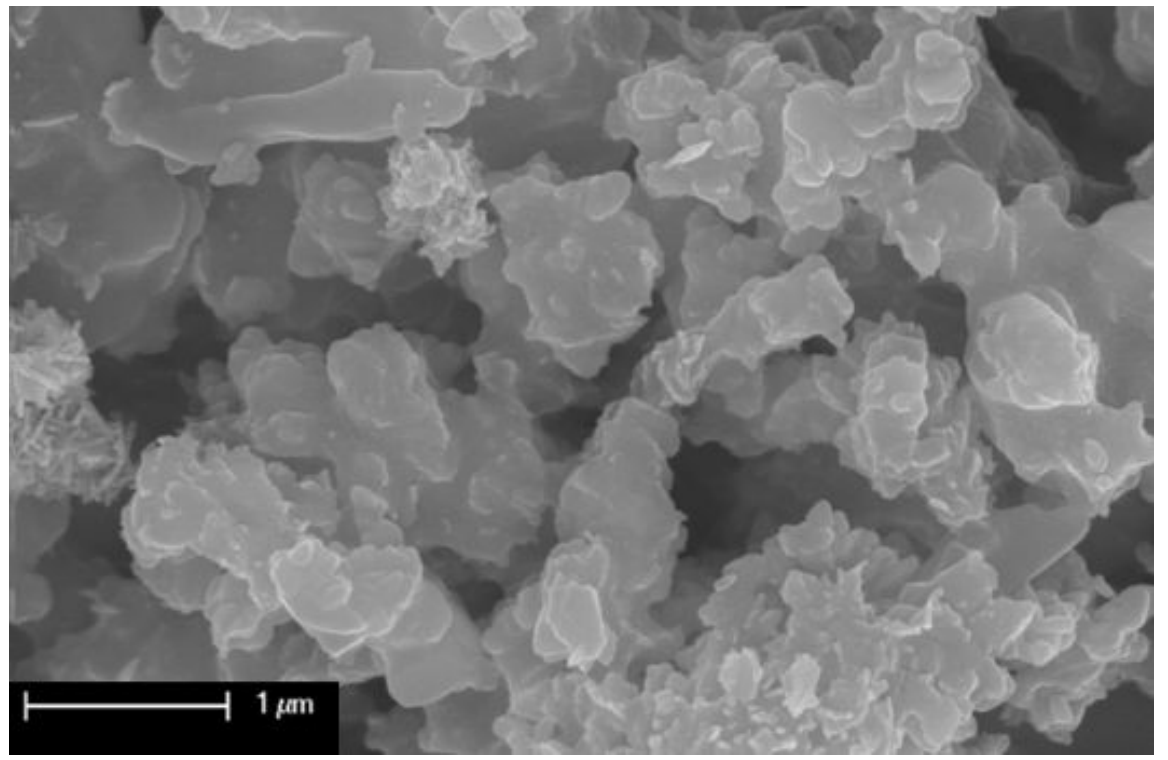

Figure S5. Additional SEM images of 2D@Ag.

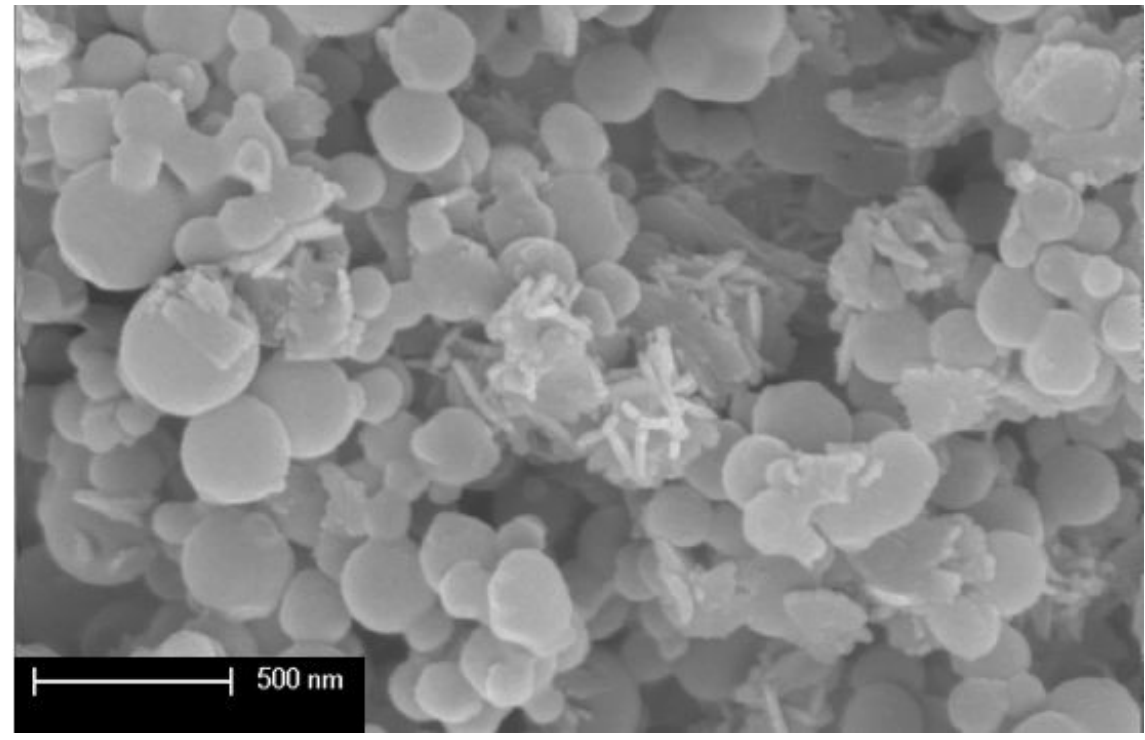

Figure S6. Additional SEM images of 2D@Cu. 


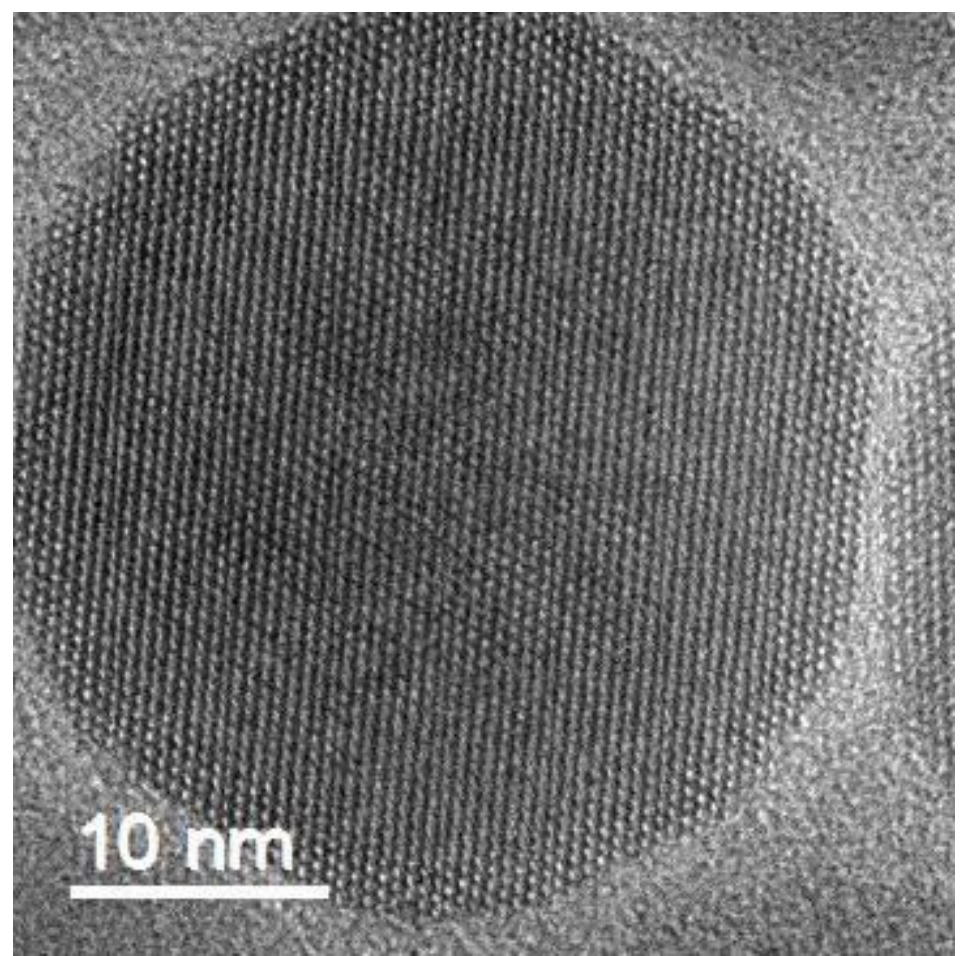

Figure S7. HRTEM photographs of $\mathrm{Yb}^{3+}$ sensitized hexagonal-phase UCNPs.

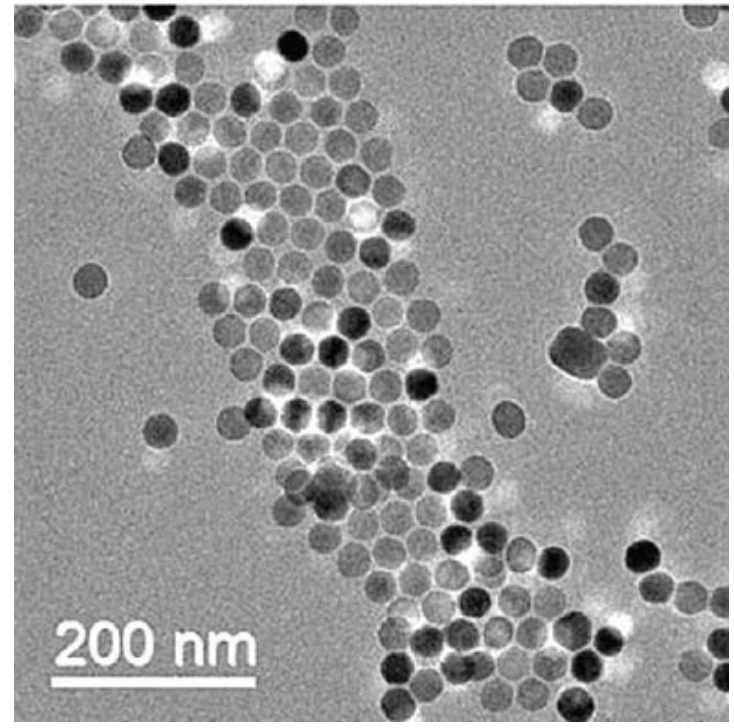

Figure S8. The TEM image of $\mathrm{Yb}^{3+}$ sensitized hexagonal-phase UCNPs. 

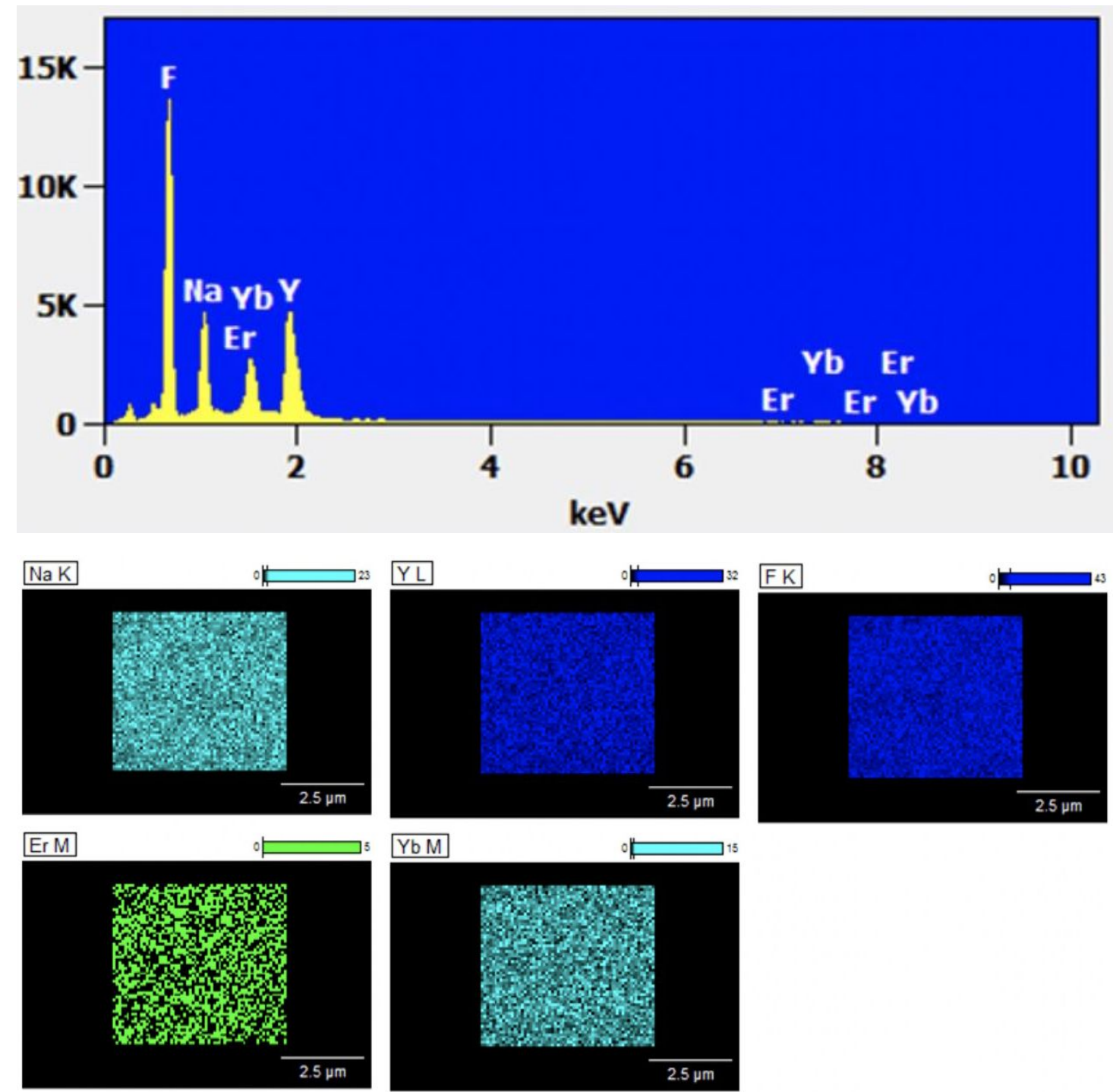

Figure S9. The EDXA analysis of $\mathrm{Yb}^{3+}$ sensitized hexagonal-phase UCNPs. 


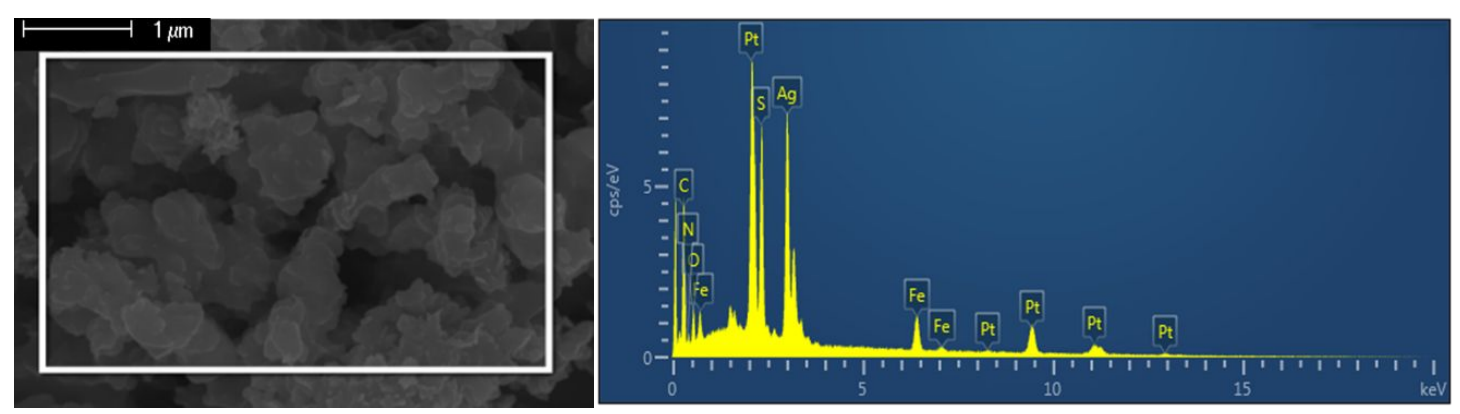

Figure S10. The EDX analysis of 2D@Ag.

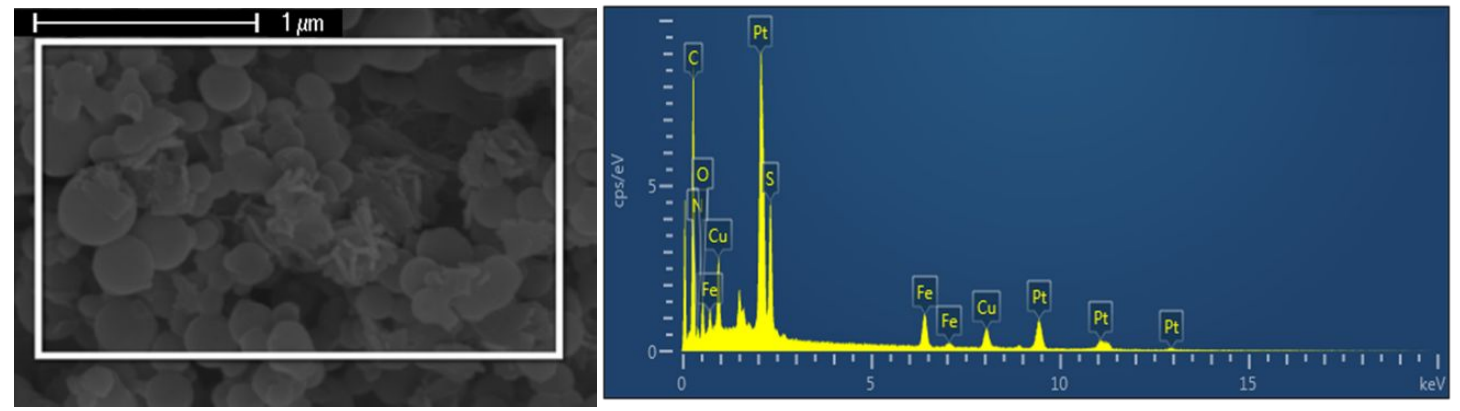

Figure S11. The EDX analysis of 2D@Cu. 